\title{
Spatial distribution of growth hormone receptor, insulin-like growth factor-I receptor and apoptotic chondrocytes during growth plate development
}

\author{
J Cruickshank, D I Grossman, R K Peng, T R Famula \\ and A M Oberbauer \\ Department of Animal Science, University of California, One Shields Avenue, Davis, California 95616, USA \\ (Requests for offprints should be addressed to A M Oberbauer; Email: amoberbauer@ucdavis.edu)
}

\begin{abstract}
Linear bone growth depends upon proliferation, maturation, and apoptosis of growth plate chondrocytes, processes regulated by growth hormone $(\mathrm{GH})$ and insulin-like growth factor-I (IGF-I). To investigate the contribution of GH, IGF-I and apoptosis to growth plate function, the expression of GH receptor (GHR) and IGF-I receptor (IGF-IR) mRNA were evaluated by in situ hybridization in fractionated costochondral growth plates of growing rats (at 2, 4, and 7 weeks). Apoptosis was determined by TUNEL assay and morphology in histological sections. GHR mRNA was greatest in resting cells with hypertropic cells increasing GHR expression with increasing age. Hypertropic and resting cell IGF-IR mRNA declined over the ages studied. Receptor mRNA expression was altered by exposing cells to GH or IGF-I. GH and IGF significantly decreased GHR mRNA in proliferative cells. GH and IGF also decreased IGF-IR mRNA in
\end{abstract}

resting cells and the 2- and 4-week-old proliferative and hypertropic cells. Treating cells in culture with GH increased the number of apoptotic cells across all ages and zones. Histologically, apoptotic cells were observed at the chondro-osseous junction and within actively proliferating chondrocytes but not in resting cells. Apoptosis was highest at 4 weeks of age with lateral regions displaying the greatest number of cells undergoing apoptosis. These data indicate that apoptosis plays a role in growth plate function, particularly spatial configuration as indicated by the preferential lateral cell apoptosis. The susceptibility of proliferative cells to GHR and IGF-IR down regulation during the period of greatest apoptosis supports a role for the GH-IGF axis in both proliferation and apoptosis during growth plate development.

Journal of Endocrinology (2005) 184, 543-553

\section{Introduction}

Linear bone growth results from proliferation, hypertropy, and matrix production of cartilage cells in the growth plate, at whose metaphyseal end bone replaces the cartilage. Characterizing the activities of the growth plate chondrocyte in all its stages contributes to the understanding of skeletal growth and the determinants of mature body size. The growth plate of endochondral bones is comprised of cells representing various states of differentiation: the resting zone, the proliferative zone with actively dividing cells, and the hypertropic zone with maturing cells enlarging and ultimately dying. These proliferative and maturation processes are induced by numerous systemic endocrine effectors such as circulating growth hormone $(\mathrm{GH})$ or steroids. The effects of $\mathrm{GH}$ are mediated through the actions of insulin-like growth factor-I (IGF-I), a peptide growth factor produced in great abundance by hepatic tissue (reviewed in Le Roith et al. 2001). Growth hormone receptor (GHR) mRNA
(Oberbauer \& Peng 1995a) and immunologically reactive GHR (Werther et al. 1993) have also been localized to discrete regions of the growth plate, demonstrating that growth plate chondrocytes are capable of responding directly to $\mathrm{GH}$ in a paracrine manner.

Cellular proliferation and apoptosis are carefully coordinated processes within the growth plate. The GH axis is known to play a critical role in growth plate proliferation and IGF-I is an identified regulator of apoptosis in many tissues (Grimberg \& Cohen 2000, Murray et al. 2003). The interplay of proliferation and apoptosis within the developing growth plate has only recently been investigated and has centered on the hypertropic chondrocytes known to undergo apoptosis (Farnum \& Wilsman 1987, Lewinson \& Silbermann 1992, Gibson et al. 1995, Zenmyo et al. 1996, Roach 1997, Chrysis et al. 2002). The present study was designed to evaluate the involvement of apoptosis throughout the growth plate and assess the effects of GH and IGF-I on their receptors to suggest potential mediators of apoptosis. 
Utilizing growth plate chondrocytes fractionated into relatively pure populations of the three in vivo positional cellular phenotypes (Oberbauer \& Peng 1995a), we characterized the inherent ability of the resting, proliferative, and hypertropic zones to respond to the predominant endocrine/paracrine effectors. Further, we described the incidence of apoptosis in growth plate chondrocytes during development. Determining which cells undergo apoptosis at particular stages in their maturation is integral to understanding the dynamics of the growth plate.

\section{Materials and Methods}

\section{Cell culture}

Growth plate chondrocytes were isolated into the resting, proliferative and hypertropic stages of differentiation by discontinuous Percoll gradient centrifugation using a protocol modified from that previously described (Oberbauer \& Peng 1995a). Costochondral growth plates from Sprague-Dawley rats (Simonsen Laboratories, Gilroy, CA, USA) aged 2, 4 and 7 weeks were sterilely separated at the junction between the growth plate and the rib by visual dissection. This junction presents as a slight indentation in the cartilage and has been verified by evaluation of histological sections. Similarly, the distal growth plate was dissected from the calcified rib producing $\sim 2.5 \mathrm{~mm}$ growth plates. The growth plates were further sterilely dissected free of adherent tissue, minced to $1 \mathrm{~mm}$ segments and predigested by incubation in Dulbecco's modified Eagle's medium (DMEM)/F12 (Gibco BRL, Grand Island, NY, USA) containing 0.05\% trypsin (JRH Biosciences, Lenexa, KS, USA), 0.5\% hyaluronidase (Worthington Biochemical, Freehold, NJ, USA) and 2\% fetal bovine serum (FBS) (Gibco) at $37^{\circ} \mathrm{C}$ for $45 \mathrm{~min}$. The growth plates were then digested in DMEM/F12 containing $0.3 \%$ collagenase type II (Worthington) and 2\% FBS at $37^{\circ} \mathrm{C}$ for $30 \mathrm{~min}$. The released tissue debris and freed cells were discarded and fresh collagenase reagents were added and the incubation continued for an additional $4 \cdot 5 \mathrm{~h}$. All incubations were carried out with gentle rocking. After enzymatic digestion of the extracellular matrix, the cells were purified by filtration through a 70-micrometer nylon cell strainer (Becton Dickinson, Franklin Lakes, NJ, USA). The cells were centrifuged at $200 \boldsymbol{g}$ for $5 \mathrm{~min}$, washed in DMEM/F12 twice and resuspended in $1 \mathrm{ml}$ DMEM/F12 medium.

The chondrocytes were purified by density gradient fractionation as previously described (Oberbauer \& Peng 1995a). Briefly, following the digestion to release the chondrocytes, cells were fractionated by layering on a discontinuous isotonic Percoll (Pharmacia, Piscataway, NJ, USA) gradient prepared by weight $(1 \cdot 0250,1 \cdot 0371$, and $1 \cdot 0481 \mathrm{~g} / \mathrm{ml})$. Three fractions, representing hypertropic, proliferative and resting zone cells, were isolated and counted after centrifugation (15 $\mathrm{min}, 1875 \mathrm{~g}, 20^{\circ} \mathrm{C}$ ).
Cellular morphology was evaluated to confirm that each fraction contained the expected cell type. Fraction I, the topmost fraction, contained hypertropic cells, which were large, round highly vacuolated cells. Fraction II contained proliferative cells which were morphologically smaller than the cells in fraction I and contained a prominent nucleolus and large nucleus. The final fraction contained small round cells with little cytoplasm representing resting cells. We have previously verified the cell types derived by density gradient isolation (Oberbauer \& Peng 1995a).

Cells were rinsed in DMEM/F12 media (Gibco) supplemented with 1\% FBS (Gibco) and incubated for $6 \mathrm{~h}$ in a suspension culture with $1 \%$ FBS-supplemented media (control), 1\% FBS-supplemented media with $30 \mathrm{ng} / \mathrm{ml}$ GH (a gift from Monsanto, St Louis, MO, USA) added, or $1 \%$ FBS-supplemented media with $50 \mathrm{ng} / \mathrm{ml} \mathrm{IGF-I}$ (Collaborative Research, Bedford, MA, USA) added. At the conclusion of a 6-h incubation, cells were washed twice in serum-free media and then fixed for $10 \mathrm{~min}$ on ice in a $4 \%$ paraformaldehyde, phosphate buffered saline (PBS) solution. Cells were centrifuged, brought up in 4\% paraformaldehyde-PBS and an aliquot was spread on poly-L-lysine-coated slides. Once the spread had dried, the attached cells were fixed in 4\% paraformaldehyde-PBS for $1 \mathrm{~min}$. Separate fixed cell slide preparations from growth plate cells cultured with vehicle, GH, or IGF-I were used for either the detection of growth hormone receptor (GHR) and insulin-like growth factor I receptor (IGF-IR) mRNA or for the detection of apoptosis described below.

The GHR cDNA was a generous gift from Dr F Talamantes (University of California, Santa Cruz, CA, USA) and the IGF-IR cDNA was obtained from the American Type Culture Collection (Rockville, MD, USA). Probes were labeled with fluorescein-dUTP in a random priming reaction and detected with antifluorescein alkaline phosphatase conjugate. All reagents for the probe labeling and detection were purchased from Amersham (Arlington Heights, IL, USA). Probe generation, hybridization, and detection of probes to the fixed growth plate chondrocytes on the poly-L-lysine slides were as published (Durrant et al. 1995). Briefly, slides were incubated in $0.02 \mathrm{M} \mathrm{HCl}$ for $10 \mathrm{~min}$ followed by a $90-\mathrm{s}$ wash in $0.02 \%$ Triton $\mathrm{X}-100$. The slides were washed twice in PBS and incubated at $37^{\circ} \mathrm{C}$ with $25 \mu \mathrm{g} / \mathrm{ml}$ proteinase $\mathrm{K}$. The proteinase $\mathrm{K}$ was inactivated by washing in $0.2 \%$ glycine for $5 \mathrm{~min}$ at room temperature; this was followed by a 15 -min wash in $20 \%$ cold acetic acid and slides were re-equilibrated in 2 washes of PBS. The denatured probe was added to $50 \%$ formamide hybridization buffer $(50 \%$ deionized formamide, $10 \%(\mathrm{w} / \mathrm{v})$ dextran sulfate, $1 \times$ Denhardt's solution and $200 \mu \mathrm{g} / \mathrm{ml} \mathrm{tRNA}$ in $0 \cdot 6 \mathrm{M} \mathrm{NaCl}, 10 \mathrm{mM}$ Tris- $\mathrm{HCl}, \mathrm{pH}$ 7.4, $1 \mathrm{mM}$ EDTA, $\mathrm{pH} \mathrm{8.0)} \mathrm{and} \mathrm{then} \mathrm{overlaid} \mathrm{on} \mathrm{the} \mathrm{growth} \mathrm{plate} \mathrm{chondro-}$ cytes. The probe was incubated overnight at $42^{\circ} \mathrm{C}$ with humidity. Unhybridized probe was removed by washing with SSC solutions of increasing stringency and finally 
rinsed with PBS. The slides were then incubated with the anti-fluorescein antibody conjugated to alkaline phosphatase, washed again, and the detection substrate added according to the manufacturer's instructions. Cells exhibiting visible colored precipitate indicated hybridization of the probe to the target GHR or IGF-IR mRNA. Negative control slides (no probe added) were included to account for nonspecific binding and no visible precipitate was observed. Apoptosis was evaluated in separate slide preparations by in situ terminal deoxynucleotidyl transferase (TdT)-mediated deoxy-UTP nick-end labeling (TUNEL) assay used according to the manufacturer's instructions (DeadEnd colorimetric apoptosis detection system, Promega, Madison, WI, USA).

\section{Histology}

Costochondral growth plates were dissected rapidly from male Sprague-Dawley rats aged 2, 4 and 7 weeks. Extraneous tissue was trimmed from the growth plate leaving intact the growth plate's three zones, the metaphyseal bone directly adjoining the hypertropic zone, and the cartilage tissue directly epiphyseal of the resting zone. The dissected growth plates were fixed for 2 to $3 \mathrm{~h}$ in $2 \%$ paraformaldehyde- $2 \%$ glutaraldehyde in $0.05 \mathrm{M}$ cacodylate buffer, $\mathrm{pH} 7 \cdot 3$ with $0 \cdot 7 \%$ ruthenium hexamine trichloride (RHT) (Polysciences Inc., Warrington, PA, USA) at room temperature; they were then rinsed in cacodylate buffer (Breur et al. 1991) followed by a 2-h rinse in $70 \%$ ethanol. Tissue dehydration and processing was carried out in a VIP processor (Tissue-Tek VIP 1000/2000, Miles Scientific, Naperville, IL, USA). Following paraffin embedding, the growth plates were cut in 3-micron sections transverse to the long axis with a rotary microtome (LKB Bromma). Serially cut sections were stained with $1 \%$ toluidine blue. Stained sections were viewed under a light microscope at magnifications up to $1250 \times$ and photographed with Kodacolor 200 film (Eastman Kodak, Rochester, NY, USA).

The entire growth plate from two to three rats of each age group was analyzed for the proportion of proliferative and hypertropic cells undergoing apoptosis. Cells were determined to be apoptotic if they exhibited morphological features typical of cells undergoing apoptosis including overall cellular shrinking, nuclear and cytoplasmic condensation, and plasma membrane blebbing (Wyllie 1987). The entire growth plate was serially sectioned, and morphologically apoptotic and normal cells (a cell being defined as a cell profile with a discernible nucleus) were counted in each serial section across the entire growth plate for each rat. The number of cells exhibiting apoptotic morphology in each section was recorded and expressed as a proportion of the total number of cells for that section. Thus, in the serial section counts, rather than attempting to follow a single cell through adjacent sections, we determined the total proportion of cells exhibiting apoptotic morphology within the entire growth plate. Therefore, by counting every cell in every section the true proportion of cells undergoing apoptosis in a growth plate was generated, essentially a 3-D representation of the apoptotic events of the growth plate. The morphological assessment and relative proportion of apoptotic cells within growth plate sections were corroborated by counting cells labeled by the in situ TUNEL assay (DeadEnd colorimetric apoptosis detection system, Promega) on multiple, though not serial sections, from additional age-matched rats.

The growth plates of the fourth left rib of a single 4-week-old rat was fixed as above and then in a secondary fixative of $1 \%$ osmium tetroxide in cacodylate buffer with $0 \cdot 7 \%$ RHT for transmission electron microscopy. The fixed tissues were rinsed with water, dehydrated in graded acetones, then embedded in epoxy resin. Several $1-\mathrm{mm}$ sections were cut and stained with azure II and methylene blue. From selected areas, ultrathin $(60-90 \mathrm{~nm})$ sections were cut and placed on 200 mesh copper grids without support, and stained with uranyl acetate and lead citrate. The grids were viewed in a Phillips EM-400 transmission electron microscope.

\section{Data analysis}

Within each growth plate fraction, two hundred cells from each of four independent fields were evaluated for in situ hybridization to GHR or IGF-IR mRNA. A field was chosen and all cells were counted left to right, top to bottom until data from 200 cells were recorded. The number of cells displaying label, that is a visible colored precipitate indicating hybridization to the target mRNA, was recorded as a percentage of the 200 cells for each region counted on each slide. The percentage of labeled cells within each fraction was analyzed by the least squares analyses of variance procedures using PROC GLM of SAS (SAS 2001) with fixed effects of age, treatment, fraction, and their interaction. The complete experiment of cell isolation and fractionation, culture, treatment, and hybridization was carried out twice at different times using 4-6 rats at each age. This same approach was applied to the cultured cells used in TUNEL labeling except that 1000 cells were counted per slide. A single individual evaluated all in situ slides without the treatments being revealed at the time of scoring. Likewise, a single individual evaluated the fixed cells for TUNEL labeling and the histological slides for apoptotic morphology and TUNEL labeling; treatment groups and ages were not disclosed to the individual during scoring.

Histological data for the cellular profiles throughout the entire plate were expressed as a percentage of apoptotic cells relative to the total number of chondrocytes in the hypertropic and proliferative zones and in the central and lateral regions of the growth plate. Percentage data were also evaluated through the angular transformation to ensure residuals were normally distributed 


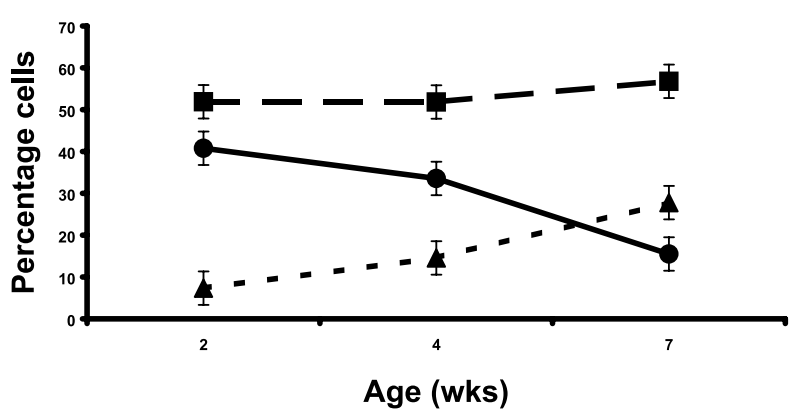

Figure 1 Cell types within the rat growth plate. Resting (circles), proliferative (squares), and hypertropic (triangles) cells as a proportion of total growth plate cells are shown as a function of age in fractionated chondrocytes, without distinction made as to live or apoptotic cells. Values are expressed as means \pm S.E.M

(Snedecor \& Cochran 1967). Both original-scale and transformed data were evaluated with models that included fixed effects for age and location in an analysis of variance through PROC GLM of SAS (SAS 2001). A term for the animal providing the data point was also included as part of a mixed model analysis (using PROC MIXED, SAS). Residuals were analyzed through PROC UNIVARIATE of SAS, and the Shapiro-Wilk statistic, W, was considered as a test of normally distributed residuals (Shapiro \& Wilk 1965).

\section{Results}

\section{Fractionated chondrocytes}

While the absolute numbers of growth plate cells remained relatively constant over the growth period examined, the proportion of cells residing in each of the three zones of the growth plate changed with age (Fig. 1). The proportion of resting zone cells declined with age $(P<0 \cdot 01)$ while hypertropic cells became more abundant with increasing age $(P<0 \cdot 01)$ nearly matching the decrease observed for resting cells. The proportion of proliferative zone cells was similar across the ages studied.

In untreated cells, GHR levels were a function of growth plate cell type as well as age of the animal (Table $1)$. When taken as a whole and averaged across the ages studied, the greatest average GHR mRNA levels $(77 \cdot 1 \pm 1 \cdot 0 \%$ of cells positively expressing GHR mRNA) were found in the resting cells with a precipitous decline in expression across the growth plate cells $(51 \cdot 2 \pm 1 \cdot 0 \%$ and $34 \cdot 8 \pm 1 \cdot 0 \%$ positively expressing GHR in proliferative and hypertropic cells respectively, $P<0 \cdot 05)$. As the animals aged, the relative proportion of cells positively labeled for GHR mRNA increased in the resting and hypertropic zones $(P<0 \cdot 05)$.

Likewise, IGF-IR mRNA expression was influenced by cell position within the growth plate and the age of the
Table 1 Percentage of growth plate chondrocytes in each fractionated zone at the three ages studied that express GHR and IGF-IR mRNA as determined by in situ hybridization. Values are presented as means \pm S.E.M.

\begin{tabular}{|c|c|c|c|}
\hline & Age (wks) & GHR & IGF-IR \\
\hline \multicolumn{4}{|l|}{ Zone } \\
\hline \multirow[t]{3}{*}{ Resting } & 2 & $72 \cdot 0 \pm 1 \cdot 7^{a}$ & $78 \cdot 5 \pm 2 \cdot 1^{b}$ \\
\hline & 4 & $76 \cdot 3 \pm 1 \cdot 7^{a}$ & $76 \cdot 1 \pm 2 \cdot 1^{b}$ \\
\hline & 7 & $83 \cdot 1 \pm 1 \cdot 7^{b}$ & $66 \cdot 6 \pm 2 \cdot 1^{a}$ \\
\hline \multirow[t]{3}{*}{ Proliferative } & 2 & $50 \cdot 5 \pm 1 \cdot 7$ & $27 \cdot 1 \pm 2 \cdot 1^{a}$ \\
\hline & 4 & $51 \cdot 3 \pm 1 \cdot 7$ & $44 \cdot 1 \pm 2 \cdot 1^{b}$ \\
\hline & 7 & $51 \cdot 9 \pm 1 \cdot 7$ & $28 \cdot 9 \pm 2 \cdot 1^{a}$ \\
\hline \multirow[t]{3}{*}{ Hypertropic } & 2 & $33 \cdot 3 \pm 1 \cdot 7^{a}$ & $46 \cdot 1 \pm 2 \cdot 1^{b}$ \\
\hline & 4 & $31 \cdot 0 \pm 1 \cdot 7^{a}$ & $32 \cdot 0 \pm 2 \cdot 1^{\mathrm{a}}$ \\
\hline & 7 & $40 \cdot 3 \pm 1 \cdot 7^{b}$ & $33 \cdot 9 \pm 2 \cdot 1^{a}$ \\
\hline
\end{tabular}

Means for each receptor mRNA within a cell zone designated with different superscripts are significantly different across age $(P<0 \cdot 05)$.

animal (Table 1). Resting zone cells initially expressed high levels of IGF-IR mRNA which decreased with increasing age, while proliferative zone cell IGF-IR expression transiently increased at 4 weeks of age. The hypertropic zone cells exhibited a significant depression in the level of IGF-IR between weeks 2 and 4 which persisted through week $7(P<0 \cdot 05)$.

Treatment of the cells with GH or IGF-I significantly altered the proportion of cells expressing GHR mRNA. In general, at early ages GH increased its GHR expression in the resting cells and decreased its GHR expression in the proliferating cells $(P<0 \cdot 05)$ relative to the untreated cells. Figure 2 illustrates the change in expression of GHR mRNA as a consequence of treating the cells with GH or IGF-I. GH had no effect on hypertropic cell GHR levels except at 4 weeks of age when GH treatment significantly increased GHR expression. Treating cells with IGF-I induced no change in GHR for resting cells, greatly reduced GHR in 2- and 4-week-old proliferative cells $(P<0 \cdot 05)$, and reduced expression in 7-week-old hypertropic cells. In contrast, GH treatment had little effect on proliferative cell IGF-IR expression, while dramatically reducing IGF-IR in resting cells (Fig. 3). The effects of $\mathrm{GH}$ on IGF-IR expression in hypertropic cells was age dependent: at 2 weeks of age, GH down regulated IGF-IR expression but at 4 weeks of age, GH increased IGF-IR mRNA. IGF-I treatment significantly depressed IGF-IR mRNA levels at all ages in the resting zone, at 2 and 4 weeks in the proliferative zone although only significantly $(P<0 \cdot 05)$ at 4 weeks, and in 2 -week-old hypertropic cells $(P<0 \cdot 05)$.

When fractionated chondrocytes were evaluated for apoptosis by TUNEL assay, the number of positively labeled cells was a function of age and fraction $(P<0 \cdot 05)$. The greatest number of apoptotic cells $(256 \cdot 2 \pm 24 \cdot 5)$ was 


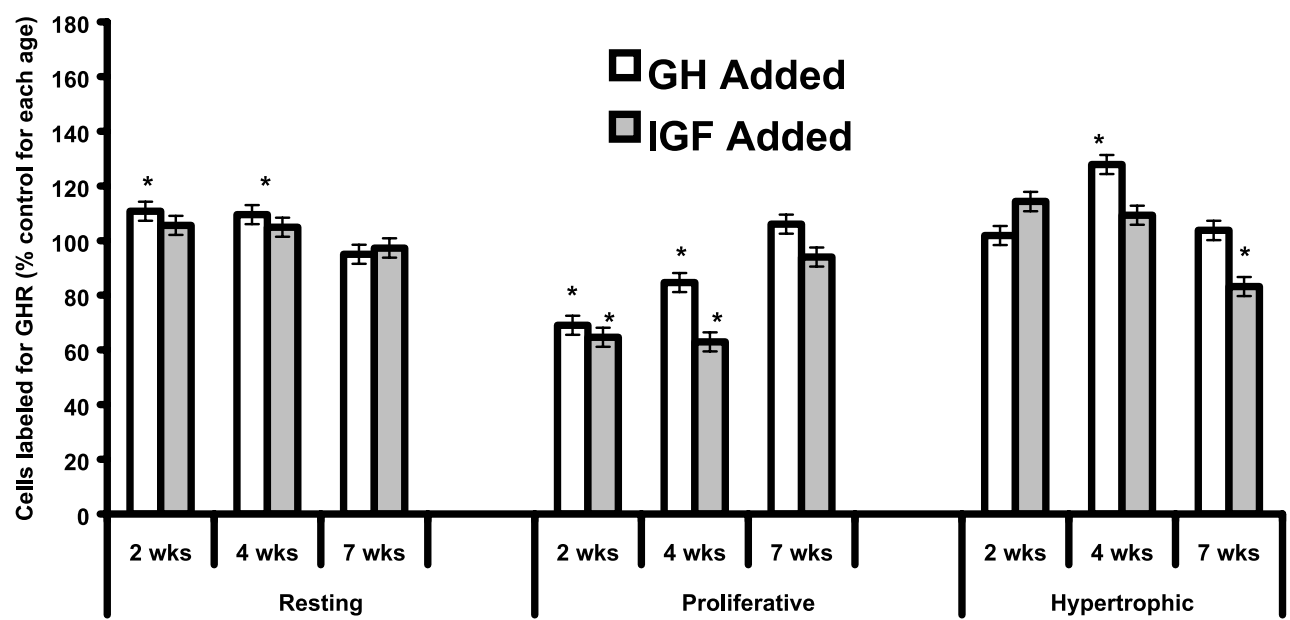

Figure 2 Growth hormone receptor mRNA distribution in fractionated growth plate cells as a function of age and GH (open bars) or IGF-I exposure (filled bars). The data are presented as the mean percentage $( \pm$ S.E.M) of the GHR mRNA in untreated cells for each age and fraction. Within a given age, bars with an asterisk are significantly different from $100 \%(P<0 \cdot 05)$, indicating that the $\mathrm{GH}$ or IGF-I treatment altered mRNA expression relative to untreated control cultures.

seen in the resting zone of 7 -week-old rats. All other zones and ages had equivalent numbers of TUNEL-positive cells $(127 \cdot 8 \pm 24 \cdot 5)$ which were significantly lower than that seen for the 7-week-old resting zone $(P<0 \cdot 05)$. Only GH affected apoptosis: treatment with $\mathrm{GH}$ resulted in an overall higher number of apoptotic cells $(P<0 \cdot 05)$ regardless of age or zone $(118 \cdot 6 \pm 20 \cdot 7,183 \cdot 4 \pm 20 \cdot 7,106 \cdot 9 \pm$ $22 \cdot 4$ for untreated, GH-treated and IGF-I-treated cells respectively). There were no significant treatment-byzone or treatment-by-age-by-zone interactions.

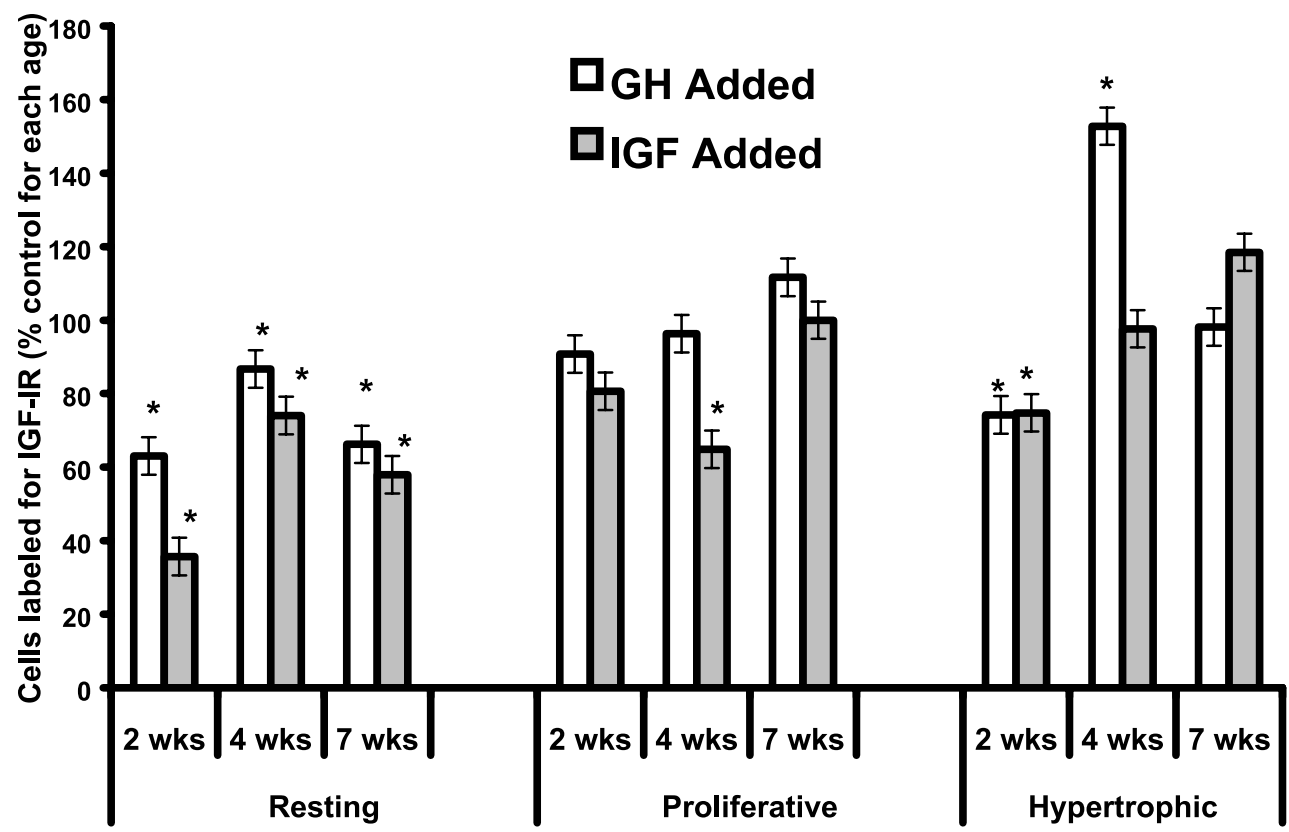

Figure 3 IGF-I receptor mRNA distribution in fractionated growth plate chondrocytes as a function of age and GH (open bars) or IGF-I (filled bars) exposure. The data are presented as the mean percentage $( \pm$ S.E.M) of the IGF-IR mRNA distribution of untreated cells for each age and fraction. Within a given age, bars with an asterisk are significantly different from $100 \%(P<0 \cdot 05)$, indicating that the GH or IGF-I treatment altered mRNA expression relative to untreated control cultures. 


\section{Histology}

In toluidine blue stained growth plate sections, some hypertropic cartilage cells at the chondro-osseous junction had a shrunken, condensed phenotype typical of the apoptotic morphology of nuclear and cytoplasmic condensation and plasma membrane blebbing; adjacent hypertropic cells appeared normal in size and shape (Fig. 4C). Furthermore, shrunken, condensed cells were scattered throughout the less mature hypertropic zone. These compact, darkly staining cells were surrounded by normal, fully hydrated cells (Fig. 4B). In some sections, cells in a single proliferative column exhibited condensed morphology indicating removal of entire regions of cells. As fixation artifacts can significantly alter cellular morphology, the RHT fixative was compared with $10 \%$ buffered formalin for the ability to retain cellular morphology. In sections from the tissue fixed with RHT, the membranes of all cells were intact with minimal cell shrinkage following fixation. Sections from the formalinfixed growth plates displayed extreme shrinkage of the chondrocytes; universally, the cell membranes were not in contact with the cartilage matrix. With formalin fixation, nearly all cells appeared morphologically shrunken, thereby making an accurate determination of a cell's viability impossible (data not shown). The visual morphological determination of apoptotic cells was corroborated by the in situ TUNEL assay (Fig. 5).

Growth plate cells in the electron photomicrographs had a very dark, condensed morphology, like that described by Farnum \& Wilsman (1987, 1989a,b). Corroborating the toluidine blue stained histological sections, some terminal and non-terminal hypertropic chondrocytes (Fig. 6) exhibited this deeply condensed morphology. In addition, a number of proliferative zone cells, both mid-column and at what appeared to be the ends of columnar cell clusters, were likewise condensed.

In both electron and light microscopy fields, darkly staining, compact cells lay adjacent to fully hydrated, viable cells, even at the last transverse septum adjoining the metaphyseal bone. As was apparent in the electron photomicrographs, the nuclei of the dark cells were intact, but the nuclear membranes were convoluted. Chromatin was condensed, but not completely marginated to the nuclear lamina. Rather, much of the chromatin was condensed in clumps that were diffuse through the nucleus. Condensed chondrocytes had cytoplasmic vacuolization and most had filopodia extending out from the cell perimeter. Observed cells exhibited very little to none of the rounded blebbing or pinching off that typify the final stages of soft tissue apoptosis. The condensed terminal hypertropic cells retracted from their pericellular matrices, clearly defining their respective lacunae. The apoptotically condensed pre and early hypertropic cells were, in contrast, in full contact with the surrounding matrix, indicative of lacunar collapse as the apoptotic cell condensed.
(A)

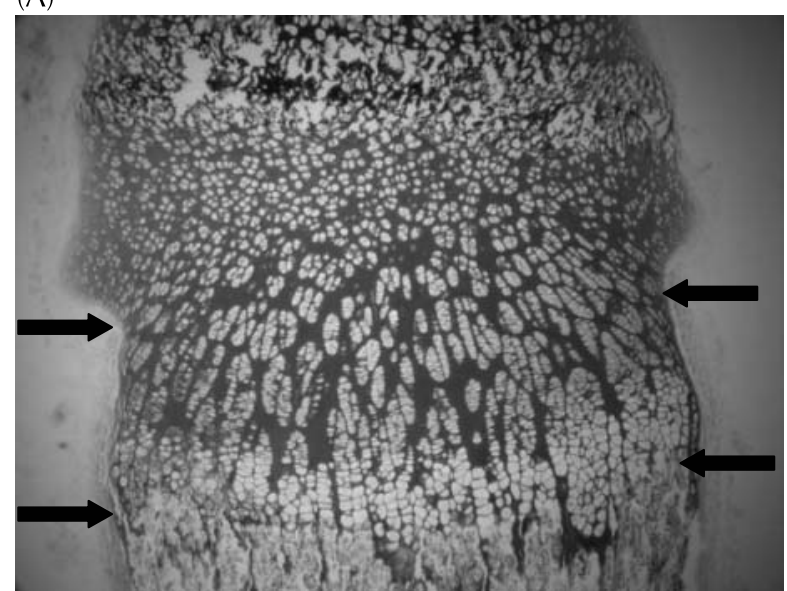

(B)

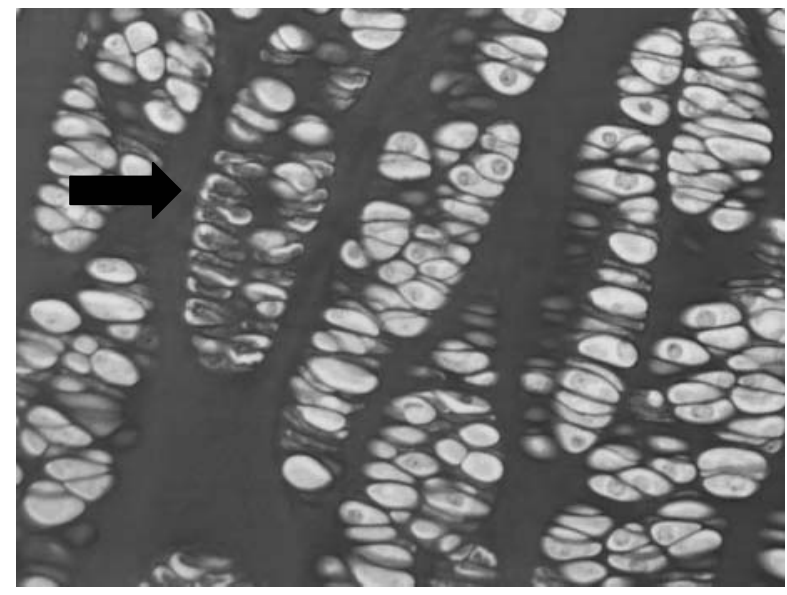

(C)

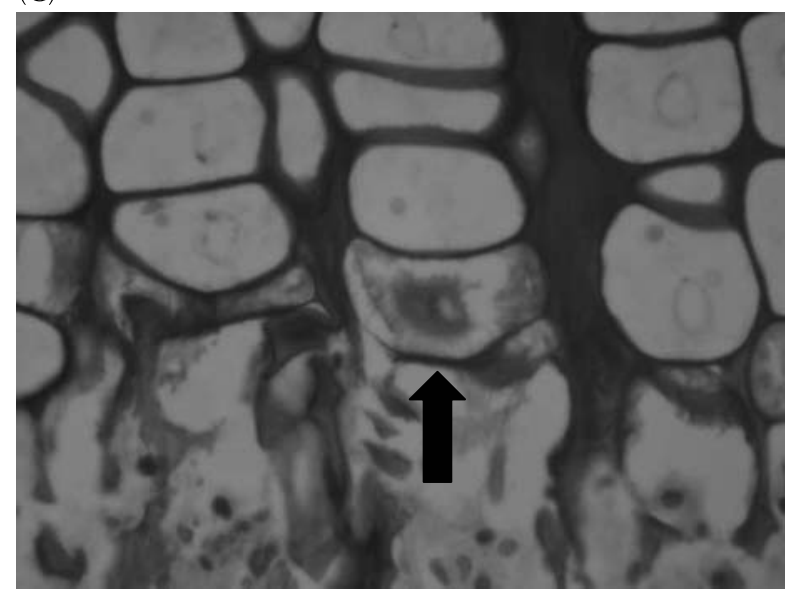

Figure 4 Toluidine blue stained 3-micron sections of 4-week-old rat costrochondral (A) entire growth plate (magnification $\times 40$ ), (B) proliferative chondrocytes (magnification $\times 200$ ) and $(C)$ hypertropic chondrocytes (magnification $\times 600$ ). Arrows in A define upper and lower ends of the growth plate, while the arrows in $\mathrm{B}$ and $\mathrm{C}$ point to morphologically apoptotic chondrocytes. Note entire columns of proliferative cells undergoing apoptosis in B. 


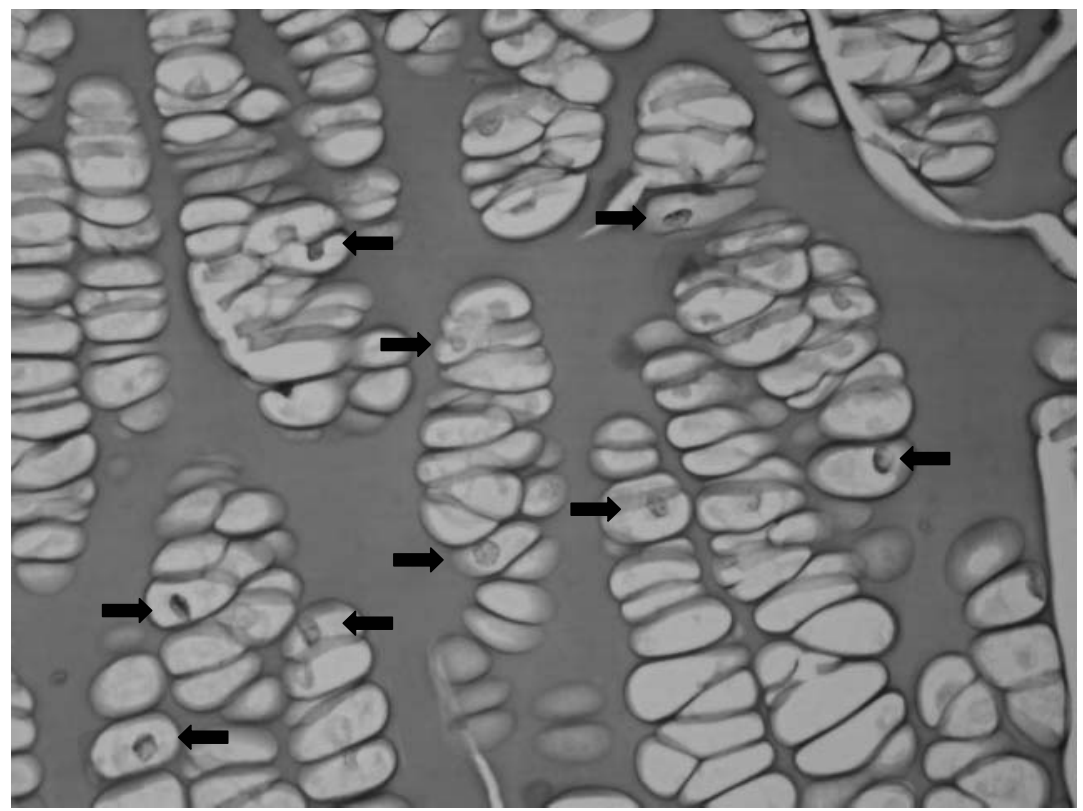

Figure 5 Apoptosis in the proliferative zone. TUNEL-positive cells in the proliferative zone of the growth plate of a 4-week-old rat; cells undergoing apoptosis as indicated by TUNEL-labeling have darkened nuclei and only some of those cells are identified by arrows in this photomicrograph. Magnification $\times 200$.

In statistically evaluating the apoptotic data, the untransformed percentage data were not normally distributed $(\mathrm{W}=0.926, P<0.0001)$ while the angular transformation was consistent with a normal distribution (W $=0.978$, $P<0 \cdot 094)$. This suggests that the interpretation of the results be based upon the angular transformed data. However, there was no difference in the generic conclusions of the data when the analyses of transformed and untransformed data were compared, revealing the well-known robustness of the analysis of variance to evaluate differences in means in the absence of normality (Scheffe 1959). Accordingly, to provide a simpler, more direct presentation of the conclusions, the untransformed percentage data are presented.

The relative abundance of apoptotic cells and their distribution in the growth plate varied with age and location (Figs 7 and 8). The interaction effects for age by growth plate zone and age by region (central or lateral) were both significant $(P<0.003)$ while the animal term was not $(P>0 \cdot 17)$. At 2 weeks of age, the same proportion of cells was undergoing apoptosis in both the proliferative and hypertropic zones. At 4 weeks of age, significantly more proliferative zone cells were undergoing apoptosis. By 7 weeks of age, the proportion of apoptotic cells detected in the proliferative zone was equivalent to that seen at 2 weeks of age. In contrast, the hypertropic zone had a significantly lower percentage of apoptotic cells at 7 weeks of age $(1 \cdot 6+0 \cdot 84 \%)$. At no point in time were apoptotic cells identified within the resting zone. Apop- totic cells were more evident in the lateral portion of the growth plate in the rapidly growing 2 - and 4-week-old rats. Greater than 19\% of the total lateral hypertropic cells exhibited apoptotic morphology as compared with $9 \cdot 1+0 \cdot 85 \%$ of the centrally located hypertropic cells. By the final age examined, 7 weeks, linear growth of these animals has virtually ceased and the abundance of all chondrocytes undergoing apoptosis was diminished.

\section{Discussion}

The age-related changes in growth plate zone proportions observed in the present study are consistent with a maturing growth plate. As endochondral bones elongate, the growth plate progresses from one with abundant 'stem' cells capable of being recruited into active proliferation to one characterized by a higher proportion of cells undergoing differentiation and maturation. Hughes \& Tanner (1970) developed maturation scores for the growing rat. The costochondral growth plate characteristics we observed are consistent with their overall bone scores. In their developmental aging scheme, our 2-week-old rats, being only 39\% mature, would be experiencing the maximum bone 'maturity score velocity'; our 7-weekold rats would be $87 \%$ mature and the growth velocity would have declined to one-fifth the velocity experienced at 2 weeks. Systemic GH has long been recognized for 

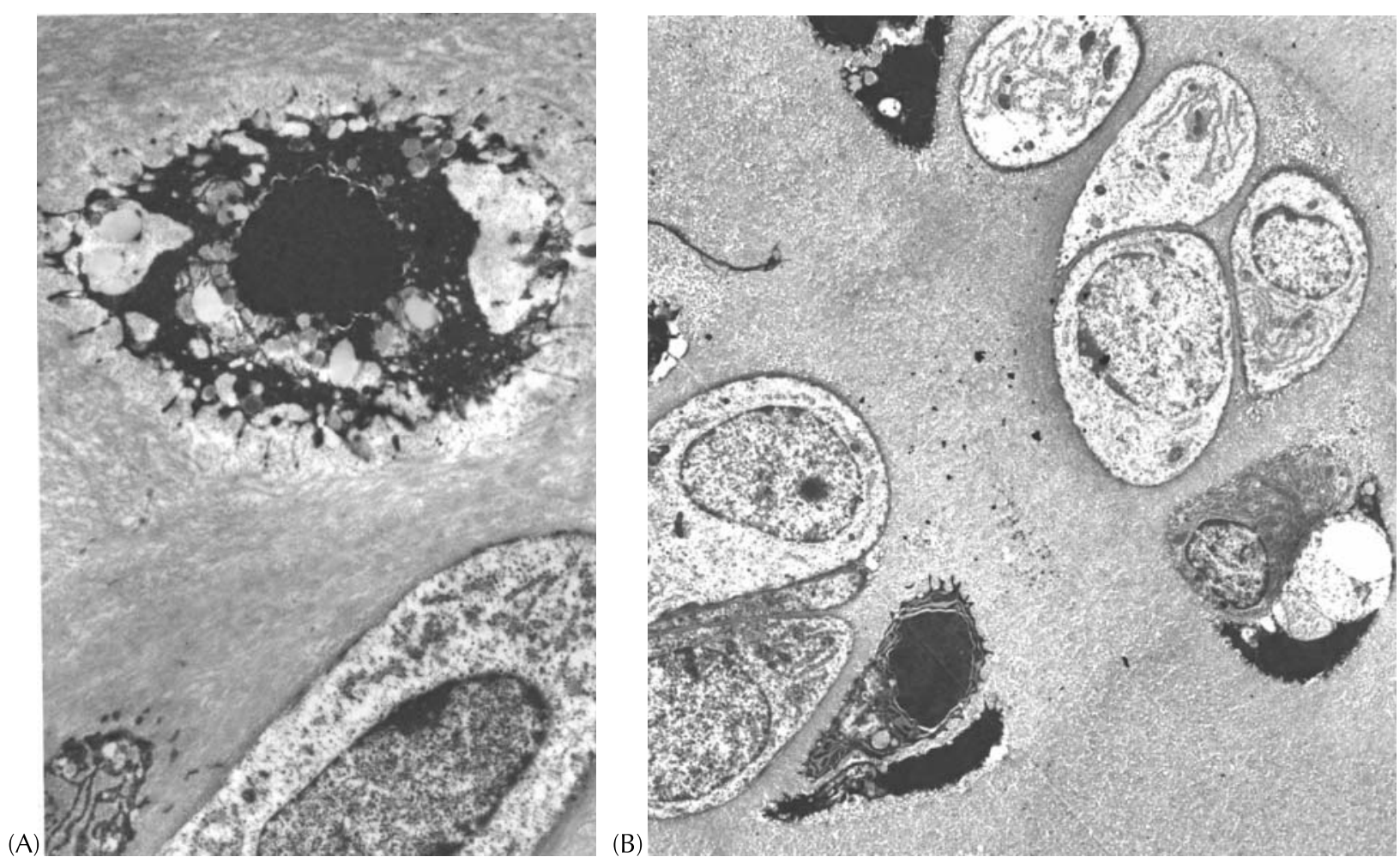

Figure 6 Apoptotic cells in the growth plate. Electron micrographs of typical condensed, darkly stained, apoptotic hypertropic (A) and proliferative (B) growth plate chondrocytes. Note the convoluted nuclear and plasma membranes, condensed chromatin, cytoplasmic vacuolization, and filopodia at the cell perimeter. A, magnification $\times 11$ 960; B, magnification $\times 4290$.

enhancing linear bone growth by altering cellular activity within the growth plate, presumably through the induction of IGF-I, thereby affecting bone growth velocity.

Elevated GH elevates circulating IGF-I as documented in animals given exogenous $\mathrm{GH}$ by injection (Davis et al. 1987), mice transgenic for the expression of GH (Chow et al. 1994), and individuals with pituitary tumors (Barreca et al. 1989). While controversy still surrounds the role of endocrine versus paracrine IGF-I in mediating GH effects, locally produced IGF-I in response to GH is well docu-

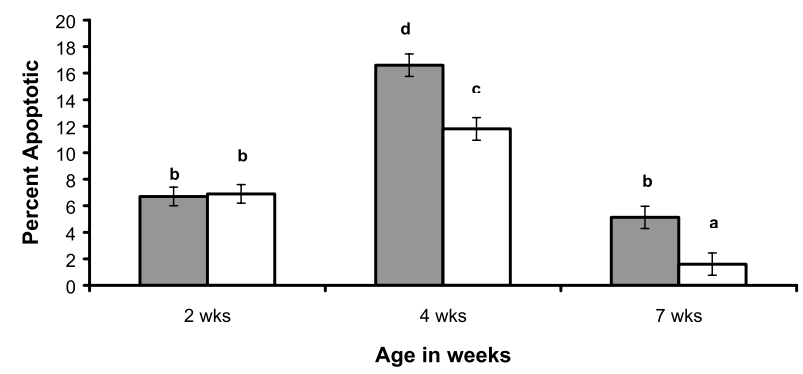

Figure 7 Apoptosis in the proliferative and hypertropic zones. Percentage of morphologically apoptotic cells in the proliferative (gray bars) and hypertropic (open bars) zones of growth plates at 2, 4 and 7 weeks of age. Different superscripts designate significant $(P<0 \cdot 01)$ differences in the means \pm S.E.M. mented (reviewed in Le Roith et al. 2001). We have previously demonstrated that application of either $\mathrm{GH}$ or IGF-I to cultured 4-week-old rat chondrocytes enhances the proliferation of cells within the proliferative zone while $\mathrm{GH}$ increases levels of IGF-IR (Oberbauer \& Peng 1995b). Those data, coupled with a restricted expression of GHR to cells of distinct phenotypes within the growth plate (Barnard et al. 1988, Werther et al. 1993, Oberbauer \& Peng 1995a), confirm a role for GH acting directly at the growth plate.

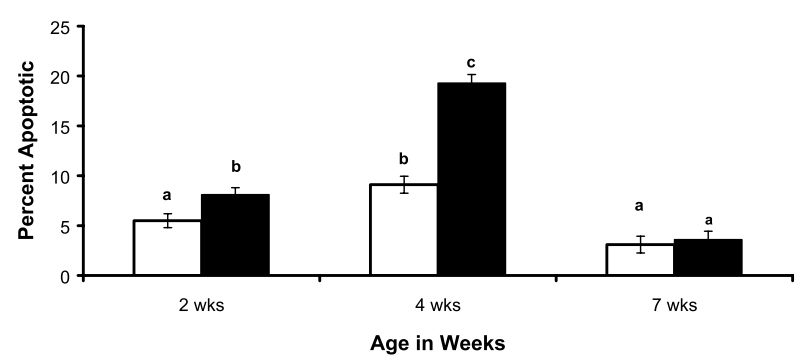

Figure 8 Apoptosis in the central and lateral growth plate regions. Percentage of morphologically apoptotic cells in the central (white bars) and lateral (black bars) regions of rat growth plates at 2, 4 and 7 weeks of age. Different superscripts designate significant $(P<0 \cdot 01)$ differences in the means \pm S.E.M. 
The distribution of GHR and IGF-IR mRNA in the zones of the growth plate changed with age in the present study. Age effects were also significant in the ability of the resting, proliferative, and hypertropic cells to respond to exogenously administered GH or IGF-I. Dependent upon the cell type within the growth plate and the age of the cell, GH was either stimulatory or inhibitory for GHR or IGF-IR mRNA expression. For example, GH stimulated the expression of GHR mRNA yet decreased IGF-IR mRNA at young ages in the resting zone. Further, at early ages, the GHR mRNA levels were reduced in the proliferative cells and IGF-IR mRNA levels were reduced in resting cells treated with either GH or IGF-I. The susceptibility of receptor mRNA expression to exogenous factors at early ages ( $2-4$ weeks) corresponds to the period of greatest overall bone growth rates (Hughes \& Tanner 1970); the consequences of this dissociation between exogenous growth factors and receptor profiles are as yet unclear, but may reflect growth factor resistance as an inherent safeguard to prevent excessive proliferation, with detrimental overgrowth of the bone. In support of this concept are studies of isolated growth plate chondrocytes in which low doses of $\mathrm{GH}(25 \mathrm{ng} / \mathrm{ml})$ were more effective at stimulating proliferation than higher doses (Oberbauer \& Peng 1995b), and studies of GH transgenic mice in which there exists a maximum limit to bone growth irrespective of the level of circulating $\mathrm{GH}$ in excess of $130 \mathrm{ng} / \mathrm{ml}$ (Oberbauer et al. 2004).

Although the vascular source supporting growth plate viability and function has not been clearly defined, the response of resting and hypertropic growth plate chondrocytes to exogenous GH by increasing GHR indicates that epiphyseal and metaphyseal vessels dually contribute to growth. The high proportion of GHR on the resting zone cells suggests that epiphyseal vascular sources of GH are involved in overall cellular proliferation and possibly growth plate closure. This supposition is strengthened by the observation that apoptosis was greatest in the 7-weekold resting cells and all zones responded to $\mathrm{GH}$ with increased apoptosis. As mentioned above, seven weeks of age represents a period of significantly decelerating bone growth characterized by severe narrowing of the majority of growth plates (Hughes \& Tanner 1970). The continued presence of high levels of both GHR and IGF-IR on resting zone cells in older animals may hasten the process as the promotion of chondrocyte proliferation (Isaksson et al. 1990, Oberbauer \& Peng 1995b) will deplete the resting cell population.

This study was also undertaken to characterize the role of apoptosis in growth plate function by ascertaining the prevalence of apoptosis beyond its known involvement in terminal hypertropic chondrocyte removal. Growth plate cell morphology, in conjunction with the TUNEL assay of both hypertropic and proliferative chondrocytes, illustrated that a portion of proliferating and mature chondrocytes within the growth plate undergo programmed cell death.
Monitoring cell morphology is an historical means of identifying apoptotic cells. The condensed, darkly staining chondrocytes observed in this study with both light and electron microscopy exhibited some, though not all, of the classic hallmarks of apoptosis. Most of the literature describing morphological changes accompanying apoptosis reflects data from 'soft' tissues like liver, thymus, and cell cultures (Wyllie 1987), which differ from the more rigid, compartmentalized environment of the growth plate. The apoptotic process may be the same with regard to proteolytic cascades, but the observable, morphological end point may differ within the growth plate matrix. In the present study, analogous to that reported by Roach \& Clarke (2000), nuclear membranes of apoptotic chondrocytes were convoluted with condensed chromatin, but no crescent-shaped chromatin patterns were seen along the inner membrane of the nucleus. The cytoplasm was condensed and vacuolized, and the plasma membranes were irregular, though no cells with fragmented nuclei or membrane blebbing were observed. The retraction of an apoptosing terminal chondrocyte from its pericellular matrix, noted in this study, is consistent with evidence from other investigators (Lewinson \& Silbermann 1992, Gibson et al. 1995). In addition, Roach \& Clarke (2000) noted similar patterns of morphological changes in growth plate chondrocytes and termed them 'dark chondrocytes' and 'paralysed chondrocytes' suggesting that these cells undergo a process of programmed cell death distinct from apoptosis.

Previously published studies of growth plates or cartilage resorption have focused on cells at the interface of cartilage and bone and have not specifically addressed apoptosis of non-terminal hypertropic and proliferative zone chondrocytes (Farnum \& Wilsman 1989a,b, Zenmyo et al. 1996, Aizawa et al. 1997), although Roach \& Clarke (2000) have reported 'dark chondrocytes' and TUNEL-positive cells within the proliferative zone. While the terminal, juxtametaphyseal apoptotic chondrocytes were retracted from their lacunae, presumably due to the mineralized interterritorial cartilage matrix, the condensed apoptotic cells located outside of the ossification front were tightly surrounded by matrix. It is logical that the pericellular matrix would remain in contact with an apoptotic cell in the proliferative and non-terminal hypertropic zones because the growth plate, in general, is a well-hydrated, flexible substrate. When an apoptotic cell condenses it would lose water to its immediate surroundings, thereby increasing the hydration of the pericellular matrix making that extracellular matrix likely to follow the shrinking cell.

As demonstrated by this work, non-terminal hypertropic cells and chondrocytes of the proliferative zone can also be removed by programmed cell death, although not likely for the same reason as terminal cells that must become apoptotic to permit the process of osteogenesis. It seems that non-terminal chondrocytes may be induced to undergo apoptosis at any time, regardless of maturity or 
position in the growth plate. Kember (1993), in his review of cell kinetics, refers to the stochastic divisions of chondrocytes in the growth plate. The programmed death of chondrocytes may also be stochastic. Such continuous disposal of cells would help maintain the appropriate shape and size of the growing bone. Chondrocytic hypertropy is the pre-eminent regulator of bone elongation (Vanky et al. 1998, Hunziker et al. 1999, Farnum et al. 2002) with chondrocytes enlarging ten to fifteen times their original volume primarily in the vertical height direction (Breur et al. 1991, Farnum et al. 2002). However, cellular width is also increased (Breur et al. 1994) as chondrocytes attain a 'spherical morphology' as they undergo hypertropy (Farquharson et al. 1999). A corresponding increase, albeit slight, in the diameter of the bone at the region of the hypertropic zone should result. The removal of selected numbers of chondrocytes by apoptosis within the proliferative zone may prevent such a distortion of the bone shape.

At four weeks of age, there were significantly more apoptotic hypertropic chondrocytes near the growth plate perimeter than in the center as indicated by the cell counts of the central and lateral fields. In some growth plates, the entire hypertropic zone portion of some cell columns were apoptotic. The great number of laterally positioned apoptotic cells at four weeks of age may serve to maintain the spatial configuration of the growth plate. This relatively high level of cell death localized to the outer growth plate chondrocytes probably contributes to final bone shape and diameter and corresponds to the period of highest growth rate in rats (Hunziker et al. 1999). Although it is possible that the lateral vs central regions differ due to fixation artifact, the presence of entire proliferative columns within the central region argues against this. In addition, Roach \& Clarke (2000) also noted whole columns of proliferative cells with morphology similar to that observed here.

Chondrocytes distant to the vasculature of the metaphyseal bone may be differently regulated with regard to their survival than those cells immediately adjacent to the forming bone. Farnum \& Wilsman (1989a) hypothesize that terminal hypertropic chondrocytes, being the only growth plate cells in the physical proximity of metaphyseal vascular signaling, receive a death-initiating stimulus from the metaphyseal vasculature through the last transverse septum. Raff (1992, 1996), however, suggests that cells compete for survival factors, and those that do not receive sufficient factors undergo programmed cell death, in this way governing the numbers of different cell types. IGF-I is one such survival factor and is produced by the cells of the growth plate. Treatment of bovine or human cartilage explants with IGF-I decreases apoptotic rates (D'Lima et al. 2001). Based on mRNA studies of growth plate chondrocytes, progressively more IGF-I is produced by cell types further from the chondro-osseous junction (Oberbauer \& Peng 1995a). IGF-IR is present in a similar gradient, with the highest level of IGF-IR mRNA in resting chondrocytes.
This seemingly complementary balance of IGF-I and IGF-IR may contribute to the survival and proliferation of growth plate cells. What causes the apoptosis of chondrocytes not immediately adjacent to the metaphyseal bone remains open to speculation. Mature hypertropic chondrocytes do not produce detectable levels of IGF-I mRNA (Oberbauer \& Peng 1995a) but increase their synthesis of IGF-IR mRNA when exposed to IGF-I. A low level of endogenous IGF-I in the hypertropic cells may contribute to their apoptosis. Likewise, during the periods of high apoptosis (4 weeks) IGF-IR levels in untreated proliferative zone cells are twofold higher than at other ages. But this IGF-IR is readily down regulated by both IGF-I and GH, suggesting a reduction in IGF-I signaling during periods of active growth controlled by GH and/or IGF-I. This reduction in IGF-I activity would diminish the ability of IGF-I to be protective against apoptosis resulting in a higher number of cells undergoing apoptosis at these ages. An additional consideration is that IGF-I resistance may affect the IGF-I activity observed in the present study. Loeser et al. (2002) reported a blunted response to IGF-I in monkeys as they aged and suggested a decrease in IGF-IR number. That would be consistent with our findings although Sakata et al. (2004) reported a disruption of intracellular IGF-IR signaling in rat bones exhibiting IGF-I resistance.

The present data indicate that apoptosis affects growth plate function beyond the known action on terminal hypertropic cell removal. Apoptosis appears to play a role in spatial configuration of the growth plate, as shown by the preferential lateral cell apoptosis. The susceptibility of proliferative cells to GHR and IGF-IR down regulation during the period of greatest apoptosis supports a role for the GH-IGF axis in the balance of proliferation and apoptosis in growth plate development.

\section{Funding}

The authors declare that there is no conflict of interest that would prejudice the impartiality of this scientific work.

\section{References}

Aizawa T, Kokubun S \& Tanaka Y 1997 Apoptosis and proliferation of growth plate chondrocytes in rabbits. Journal of Bone and Joint Surgery 79 483-486.

Barnard R, Haynes KM, Werther GA \& Waters MJ 1988 The ontogeny of growth hormone receptors in the rabbit tibia. Endocrinology 122 2562-2569.

Barreca A, Ciccarelli E, Minuto F, Bruzzi P, Giordano G \& Camanni F 1989 Insulin-like growth factor I and daily growth hormone profile in the assessment of active acromegaly. Acta Endocrinologica $120629-635$.

Breur GJ, VanEnkevort BA, Farnum CE \& Wilsman NJ 1991 Linear relationship between the volume of hypertropic chondrocytes and the rate of longitudinal bone growth in growth plates. Journal of Orthopaedic Research 9 348-359. 
Breur GJ, Turgal J, VanEnkevort BA, Farnum CE \& Wilsman NJ 1994 Stereological and serial section analysis of chondrocytic enlargement in the proximal tibial growth plate of the rat. Anatomical Record 239 255-268.

Chow JC, Murray JD, Pomp D, Baldwin RL, Calvert CC \& Oberbauer AM 1994 Regulation of insulin-like growth factor-I and binding protein-3 expression in oMt1a-oGH transgenic mice. Transgenic Research 3 127-133.

Chrysis D, Nilsson O, Ritzen EM \& Savendahl L 2002 Apoptosis is developmentally regulated in rat growth plate. Endocrine $\mathbf{1 8}$ 271-278.

Davis SR, Gluckman PD, Hart IC \& Henderson HV 1987 Effects of injecting growth hormone or thyroxine on milk production and blood plasma concentrations of insulin-like growth factors I and II in dairy cows. Journal of Endocrinology 114 17-24.

D'Lima DD, Hashimoto S, Chen PC, Lotz MK \& Colwell Jr CW 2001 Prevention of chondrocyte apoptosis. Journal of Bone and Joint Surgery 83-A (Suppl 2) 25-26.

Durrant I, Brunning S, Eccleston L, Chadwick P \& Cunningham M 1995 Fluorescein as a label for non-radioactive in situ hybridization. Histochemical Journal 27 94-99.

Farnum CE \& Wilsman NJ 1987 Morphologic stages of the terminal hypertropic chondrocyte of growth plate cartilage. Anatomical Record $219221-232$.

Farnum CE \& Wilsman NJ 1989a Cellular turnover at the chondro-osseous junction of growth plate cartilage: analysis by serial sections at the light microscopical level. Journal of Orthopaedic Research 7 654-666.

Farnum CE \& Wilsman NJ 1989 b Condensation of hypertropic chondrocytes at the chondro-osseous junction of growth plate cartilage in Yucatan swine: relationship to long bone growth. American Journal of Anatomy 186 346-358.

Farnum CE, Lee R, O'Hara K \& Urban JP 2002 Volume increase in growth plate chondrocytes during hypertropy: the contribution of organic osmolytes. Bone 30 574-581.

Farquharson C, Lester D, Seawright E, Jefferies D \& Houston B 1999 Microtubules are potential regulators of growth-plate chondrocyte differentiation and hypertropy. Bone 25 405-412.

Gibson GJ, Kohler WJ \& Schaffler MB 1995 Chondrocyte apoptosis in endochondral ossification of chick sterna. Developmental Dynamics 203 468-476.

Grimberg A \& Cohen P 2000 Role of insulin-like growth factors and their binding proteins in growth control and carcinogenesis. Journal of Cellular Physiology 183 1-9.

Hughes PCR \& Tanner JM 1970 The assessment of skeletal maturity in the growing rat. Journal of Anatomy 106 371-402.

Hunziker EB, Kapfinger E \& Saager C 1999 Hypertropy of growth plate chondrocytes in vivo is accompanied by modulations in the activity state and surface area of their cytoplasmic organelles. Histochemistry and Cell Biology 112 115-123.

Isaksson OGP, Nilsson A, Isgaard J \& Lindahl A 1990 Cartilage as a target tissue for growth hormone and insulin-like growth factor-I. Acta Paediatrica Scandinavica Supplement 367 137-141.

Kember NF 1993 Cell kinetics and the control of bone growth. Acta Paediatrica Supplement 391 61-65.

Le Roith D, Bondy C, Yakar S, Liu JL \& Butler A 2001 The somatomedin hypothesis: 2001. Endocrine Reviews 22 53-74.

Lewinson D \& Silbermann M 1992 Chondroclasts and endothelial cells collaborate in the process of cartilage resorption. Anatomical Record 233 504-514.
Loeser RF, Carlson CS, Del Carlo M \& Cole A 2002 Detection of nitrotyrosine in aging and osteoarthritic cartilage: correlation of oxidative damage with the presence of interleukin-1 beta and with chondrocyte resistance to insulin-like growth factor 1. Arthritis and Rheumatism 46 2349-2357.

Murray SA, Zheng H, Gu L \& Jim Xiao ZX 2003 IGF-1 activates p21 to inhibit UV-induced cell death. Oncogene 22 1703-1711.

Oberbauer AM \& Peng R 1995a Fractionation of growth plate chondrocytes: differential expression of IGF-I and growth hormone and IGF-I receptor mRNA in purified populations. Connective Tissue Research 31 179-187.

Oberbauer AM \& Peng R 1995 browth hormone and IGF-I stimulate cell function in distinct zones for the rat epiphyseal growth plate. Connective Tissue Research 31 188-194.

Oberbauer AM, Stiglich C, Murray JD, Keen CL, Fong DL, Smith LB \& Cushwa S 2004 Dissociation of body growth and adipose deposition effects of growth hormone in oMt1a-oGH transgenic mice. Growth Development and Aging 68 33-45.

Raff MC 1992 Social controls on cell survival and cell death. Nature 356 397-400.

Raff MC 1996 Size control: the regulation of cell numbers in animal development. Cell 86 173-175.

Roach HI 1997 New aspects of endochondral ossification in the chick: chondrocyte apoptosis, bone formation by former chondrocytes, and acid phosphatase activity in the endochondral bone matrix. Journal of Bone and Mineral Research 12 795-805.

Roach HI \& Clarke NM 2000 Physiological cell death of chondrocytes in vivo is not confined to apoptosis. New observations on the mammalian growth plate. Journal of Bone and Joint Surgery $\mathbf{8 2}$ 601-613.

Sakata T, Wang Y, Halloran BP, Elalieh HZ, Cao J \& Bikle DD 2004 Skeletal unloading induces resistance to insulin-like growth factor-I (IGF-I) by inhibiting activation of the IGF-I signaling pathways. Journal of Bone and Mineral Research 19 436-446.

SAS 2001 SAS/STAT User's Guide, Release 8·0. North Carolina: Statistical Analysis System Institute, Inc.

Scheffe H 1959 The Analysis of Variance, pp 477. New York, NY: John Wiley \& Sons.

Shapiro SS \& Wilk MB 1965 An analysis of variance test for normality (complete samples). Biometrika 52 591-611.

Snedecor GW \& Cochran WG 1967 Statistical Methods, pp 593. Ames, Iowa: Iowa State University Press.

Vanky P, Brockstedt U, Hjerpe A \& Wikstrom B 1998 Kinetic studies on epiphyseal growth cartilage in the normal mouse. Bone $\mathbf{2 2}$ 331-339.

Werther GA, Haynes K, Edmondson S, Oakes S, Buchanan CJ, Herington AC \& Waters MJ 1993 Identification of growth hormone receptors on human growth plate chondrocytes. Acta Paediatrica Supplement 391 50-53.

Wyllie AH 1987 Apoptosis: cell death in tissue regulation. Journal of Pathology 153 313-316.

Zenmyo M, Komiya S, Kawabata R, Sasaguri Y, Inoue A \& Morimatsu M 1996 Morphological and biochemical evidence for apoptosis in the terminal hypertropic chondrocytes of the growth plate. Journal of Pathology 180 430-433.

Received 5 August 2004

Accepted 23 November 2004 